\title{
Classifying Supergravity Solutions
}

\author{
Jerome P. Gauntlett \\ Blackett Laboratory, Imperial College \\ Prince Consort Rd., London, SW7 2AZ, U.K.
}

\begin{abstract}
We review the substantial progress that has been made in classifying supersymmetric solutions of supergravity theories using $G$-structures. We also review the construction of supersymmetric black rings that were discovered using the classification of $D=5$ supergravity solutions.

To appear in the Proceedings of the 37th International Symposium Ahrenshoop, "Recent Developments in String/M-Theory and Field Theory", August 23-27 2004, BerlinSchmöckwitz.
\end{abstract}

\section{Introduction}

Supersymmetric solutions of supergravity theories have played a key role in many of the most important developments in string theory. For example, supersymmetric compactifications provide a promising setting for obtaining realistic models of particle physics, a microscopic interpretation of black hole entropy in string theory is best understood for supersymmetric black holes, and various kinds of supersymmetric solutions have transformed our understanding of quantum field theory via the AdS/CFT correspondence and its generalisations.

Here we would like to review the remarkable progress that has been made over the past two and a half years in classifying such solutions. Recall that if one sets all of the matter-fields (fluxes) to zero, bosonic supersymmetric solutions must have metrics with special holonomy. Thus, the basic problem is to find the appropriate generalisation for more general solutions with non-vanishing fluxes. The key mathematical tools [1, 2] are " $G$-structures". The rough idea is to maintain the tensors arising in special holonomy manifolds, but to relax the differential conditions that they usually satisfy. For example, a Calabi-Yau three-fold has a Kähler form and a $(3,0)$ form which satisfy certain algebraic conditions. The necessary and sufficient conditions for a manifold admitting such tensors to have $S U(3)$ holonomy are that both of these forms are closed. More general $S U(3)$ structures arise by relaxing these differential conditions in a precise way.

$G$-structures have now been used to classify various kinds of supersymmetric solutions of different supergravity theories. One of the purposes of this article is to summarise what has been achieved and to highlight some remaining issues that could be examined further. Broadly speaking the work completed falls into three categories. Firstly, a classification of the most general supersymmetric solutions of supergravity theories arising as the lowenergy limit of string/M-theory. Secondly, a similar classification for simpler supergravity 
theories in lower-dimensions. And thirdly, a classification of specific classes of solutions of physical interest. For example, solutions that are products, possibly warped products, of four-dimensional Minkowski space with a six- or seven-dimensional compact internal space are of interest for phenomenological reasons, while similar products of anti-de-Sitter $(A d S)$ space with a compact internal space are of interest for the $A d S / \mathrm{CFT}$ correspondence.

Having obtained such a classification one can use the results to try and construct new solutions in explicit form. This has also been a very successful endeavour. Four prominent examples are: (i) the discovery of the maximally supersymmetric Gödel solution of $\mathrm{D}=5$ supergravity, which initiated investigations into the role of closed timelike curves in string/M-theory [3] (ii) the construction of a very rich class of geometries corresponding to $1 / 2$ BPS excitations in $A d S / C F T$ dualities [4] (iii) the discovery of an infinite class of new Sasaki-Einstein manifolds $Y^{p, q}$ that provide new $A d S_{5} \times Y^{p, q}$ solutions of type IIB supergravity [5, 6] and (iv) the discovery of supersymmetric black rings in $D=5$ supergravity, i.e., black holes with horizons of topology $S^{1} \times S^{2}$ rather than the usual $S^{3}$ [7, 8, 9, 10, 11].

The discovery of the infinite class of Sasaki-Einstein manifolds $Y^{p, q}$ was recently reviewed in 12 and so we shall not discuss them much here. However, since that review appeared there has been some very interesting developments: following some further studies of the geometry 13 the corresponding dual conformal field theories have now been identified [14] and, using the techniques of "a-maximisation" [15, the central charges of the conformal field theories have been computed [13, 16, 14] and shown to agree precisely with those predicted in [6] from a calculation of the volumes of the $Y^{p, q}$. It is worth emphasising that before the discovery of the $Y^{p, q}$ in [5, 6] and the work of [14] there were only a few $A d S_{5} / \mathrm{CFT}_{\text {examples }}{ }^{1}$ where both the geometry was explicit and the field theory was identified. Additional recent work in this area appears in [17, 18.

In this article we will briefly review the construction of the supersymmetric black ring solutions. The surprising discovery of black ring solutions was first made in a nonsupersymmetric setting in $D=5$ by Emparan and Reall in [19]. The $S^{1} \times S^{2}$ topology of the event horizon is prevented from collapsing by the rotation of the black ring. The supersymmetric generalisation, for minimal $\mathrm{D}=5$ supergravity, was found in [7] using the classification of solutions for this theory carried out in [3]. This was further generalised to black ring solutions of $\mathrm{D}=5$ supergravity coupled to vector multiplets in [9, 10, 11, and multi-concentric rings were found in [8, 11]. An interesting class of solutions occurs when there are two vector multiplets as this theory arises from a toroidal reduction of string/Mtheory. In this case the black ring solutions are specified by seven parameters and yet only carry five independent conserved charges. In other words, in marked contrast to fourdimensional black holes, these black rings violate a naive generalisation of the powerful uniqueness theorems for four-dimensional black holes. This non-uniqueness is a striking feature of the supersymmetric black rings and it will be very interesting to see how it is incorporated in a microscopic identification of the black hole entropy. Some analysis of such an identification appears in [20, 21].

\section{Classifying Supergravity Solutions}

Consider a general supergravity theory whose bosonic fields consist of a metric, $g_{\mu \nu}$, and some possible matter fields, often referred to as "fluxes", which are generically a set of $p$-form potentials for various $p$. We are only interested in bosonic solutions and so we

\footnotetext{
${ }^{1}$ Ignoring orbifolds of these examples, which are certainly interesting.
} 
set all of the fermions to zero. The equations of motion for such configurations are then, schematically,

$$
\begin{gathered}
R_{\mu \nu}-\frac{1}{2} R g_{\mu \nu}=T_{\mu \nu} \\
\text { MatterEquations, }
\end{gathered}
$$

where $T_{\mu \nu}$ is the energy momentum tensor of all the matter fields and the second line refers to the equations of motion for the matter fields and also Bianchi identities. A supersymmetric solution is one that is left invariant under certain supersymmetry transformations. Since the fermions are zero the supersymmetry variation of the bosonic fields is automatically zero and so we just need to ensure that the variation of the fermionic fields are zero. This requires that the solutions admit "Killing spinors" $\epsilon$ that satisfy

$$
\hat{\nabla}_{\mu} \epsilon=0, \quad M \epsilon=0 .
$$

Here $\hat{\nabla}$ is a connection that is schematically of the form $\hat{\nabla}=\nabla+$ fluxes $\cdot \gamma$ where $\nabla$ is the ordinary Levi-Civita connection and the remaining pieces are matter fields contracted with various anti-symmetrised products of gamma-matrices denoted generically by $\gamma$. In mathematical terms $\nabla$ is a connection on the spin bundle while, in general, $\hat{\nabla}$ is a connection on the Clifford bundle. In (2) $M$ is a matrix, that is sometimes present, that depends on the fluxes and gamma matrices and leads to additional algebraic conditions.

Now let us recall the situation when all of the matter fields (fluxes) are zero. The problem then boils down to solving

$$
R_{\mu \nu}=0, \quad \nabla_{\mu} \epsilon=0,
$$

i.e. Ricci-flat manifolds with covariantly constant spinors. Actually on a Euclidean manifold, as in a compactification manifold, the latter implies the former, but not, in general, on a Lorentzian manifold. Manifolds with covariantly constant spinors have special holonomy. In the familiar Euclidean case, Berger's classification implies that the manifold can be Calabi-Yau, with $S U(n)$ holonomy in $2 n$ dimensions, hyper-Kähler, with $S p(n)$ holonomy in $4 n$ dimensions, have $G_{2}$ holonomy in seven-dimensions and $\operatorname{Spin}(7)$ holonomy in eight dimensions. The Lorentzian case is less studied. However, some possibilities relevant for string/M-theory are discussed in [22, 23. For example, in $\mathrm{D}=11$ supergravity, the general solutions have $S U(5)$ or $\operatorname{Spin}(7) \ltimes \mathbb{R}^{9}$ holonomy.

The question therefore arises as to how to generalise this classification when the fluxes are non-zero. The key generalisation of special holonomy that we employ [1, 2] is a $G$-structure, as we discuss in the next section. Before doing that we mention that an alternative approach, analysing the "generalised holonomy" of the connection $\hat{\nabla}$ appearing in (2), has been advocated in [24]. However, as discussed in [12, this approach, so far, misses some information contained in the conditions for supersymmetry, but it is possible that it could be developed further. We also mention that an effective approach for constructing ansatze for specific sub-classes of solutions was discussed in [25]: in fact this method can also be viewed from a $G$-structure point of view.

\section{G-Structure Classification}

\section{1 $\quad G$-structures}

Let us begin with the abstract definition (see e.g. [26]). Consider an $n$-dimensional manifold $M$. The set of all frames defines the frame bundle $F(M)$ which is a principal 
$G l(n)$ bundle, consistent with the fact that an element of $G l(n)$ will transform one frame into another frame. A $G$-structure is then defined as a principal $G$ sub-bundle of $F(M)$.

In cases of interest, this definition is entirely equivalent to the existence of no-where vanishing tensors. For example, a Euclidean metric $g_{a b}$ on an $n$-dimensional manifold is equivalent to an $O(n)$ structure as one can use the metric to restrict to orthonormal frames. Similarly, if we supplement this with an orientation $\epsilon_{a_{1} \ldots a_{n}}$ we can restrict to orthonormal frames with a particular orientation and this is the data required for an $S O(n)$ structure. If we are in even-dimensions, $n=2 m$, with an almost complex structure $J_{a}{ }^{b}$, satisfying $J_{a}^{b} J_{b}{ }^{c}=-\delta_{a}^{c}$, this defines a $G l(m, \mathbb{C})$ structure. If we also have a hermitian metric this defines a $U(m)$ structure. If in addition there is an $(m, 0)$-form $\Omega$ we have an $S U(m)$ structure. While these structures are somewhat familiar, more exotic $G$-structures such as $\operatorname{Spin}(7) \ltimes \mathbb{R}^{9} \subset S O(1,10)$ also appear in classifying supergravity solutions as we shall mention later. An important feature of a $G$-structures is that it allows any tensor to be decomposed into representations of $G$.

It is important to emphasise that the existence of a $G$-structure is topological and does not entail any differential conditions on the tensors. For example, the existence of a $G l(m, \mathbb{C})$ structure, which we noted above is equivalent to the existence of a nowhere vanishing almost complex structure, does not imply that the manifold is complex, which requires additional differential conditions to be satisfied (that the Nijenhuis tensor vanishes).

That being said, there is a natural way to classify $G$-structures using differential conditions satisfied by the tensors encapsulated in the "intrinsic torsion" of the $G$-structure. For illustration consider a $G$-structure with $G \subset S O(n)$. In this case we always have a metric and hence a Levi-Civita connection $\nabla$. Roughly, one takes the covariant derivative of all the tensors $\eta$ defining the $G$-structure and then decomposes the result into irreducible $G$-modules $W_{i}$ acting on $\eta$ to obtain the intrinsic torsion:

$$
\nabla \eta \rightarrow T \in \oplus W_{i} \cong \Lambda^{1} \otimes g^{\perp}
$$

Here $\Lambda^{1}$ is the space of one-forms and $g \oplus g^{\perp}=s o(N)$ where $g$ is the Lie algebra of $G$. More precisely, and to explain the last expression, we use the fact that there is no obstruction to finding a connection $\nabla^{\prime}$ such that $\nabla^{\prime} \eta=0$ and hence $\nabla \eta=\left(\nabla-\nabla^{\prime}\right) \eta$. Define the con-torsion tensor $\left(\nabla-\nabla^{\prime}\right)$ and recall that the con-torsion has the same information as the torsion. We next note that $\left(\nabla-\nabla^{\prime}\right) \in \Lambda^{1} \otimes s o(n)$. However, since $\eta$ is $G$-invariant, only the part of the con-torsion in $\Lambda^{1} \otimes g^{\perp}$ acts on $\eta$ and this is clearly the part of the con-torsion that is independent of the choice of $\nabla^{\prime}$. This part is called the intrinsic con-torsion. The intrinsic con-torsion, or equivalently the intrinsic torsion $T$, can then be decomposed into the $G$-modules $W_{i}$. For a more detailed discussion in the physics literature see [12] and for many explicit examples see [27].

The most extreme case when all $W_{i}=0$, i.e. vanishing intrinsic torsion, is equivalent to $\nabla \eta=0$ which is equivalent to having special holonomy $G$. Thus, the intrinsic torsion is a precise measure of the deviation away from special holonomy $G$ and it is for this reason that $G$-structures are useful in classifying supergravity solutions when the fluxes are non-vanishing.

\subsection{Classifying supergravity solutions}

The classification of supersymmetric supergravity solutions has three main steps. The first two steps analyse the information contained in (2); the first is purely algebraic, while 
the second is also differential. The third step is to impose the additional conditions that the equations of motion are satisfied.

1. The first observation is that the Killing spinor $\epsilon$ defines a canonical $G$-structure ${ }^{2}$. Indeed the isotropy group of the spinor $G \subset \operatorname{Spin}(d)$ (or $\subset \operatorname{Spin}(1, d-1)$ ) is in fact a $G$-structure. In the language above, the tensors defining the $G$-structure can be simply constructed from $\epsilon$ as bi-linear differential forms, roughly of the form $\bar{\epsilon} \gamma_{(n)} \epsilon$ where $\gamma_{(n)}$ is a basis of the Clifford algebra consisting of anti-symmetrised products of $n$ gammamatrices. These tensors will satisfy a number of algebraic conditions corresponding to the $G$-structure. They can be obtained, for example, by doing various Fierz transformations.

2. The second step utilises the information that the Killing spinor $\epsilon$ satisfies the differential, and possibly algebraic, conditions in (2). After a detailed analysis one finds that the intrinsic torsion of the $G$-structure is restricted and that the fluxes are correlated with the intrinsic torsion. In general there can be components of the flux which drop out of the supersymmetry conditions (2) completely.

3. By analysing the integrability conditions for the Killing spinor equations (2), one can show that some but not all of the equations of motion are automatically satisfied [28, 2]. In general, it is sufficient to impose just the Bianchi identities and the equations of motion for the matter fields, and, in the Lorentzian case, at most one component of the Einstein's equations [2].

Typically, this classification ${ }^{3}$ provides the most general local form of the solution in terms of $G$-structure data (sometimes of a lower-dimensional manifold) as well as a number of differential conditions that remain to be solved. These techniques have now been applied in a number of different contexts. The work falls into three broad classes which we now discuss in the subsequent sections.

\section{The Most General Solutions for String/M-Theory}

The techniques described above have been used to classify the most general supersymmetric solutions of $\mathrm{D}=11$ supergravity in [2, 29]. Recall that the bosonic fields of $\mathrm{D}=11$ supergravity consist of the metric plus a four-form field strength. The most general supersymmetric solution will preserve (at least) one supersymmetry, i.e. admit a Killing spinor $\epsilon$. It turns out that there are two distinct classes of supersymmetric solutions, one with an $S U(5)$ structure and the other with a $\operatorname{Spin}(7) \ltimes \mathbb{R}^{9}$ structure, corresponding to the two possible isotropy groups of spinors of $\operatorname{Spin}(10,1)[22$. The two cases can be distinguished by the algebraic conditions satisfied by the bi-linears constructed from the spinor. From a single spinor we can construct a one-form, $K$, a two-form $\Omega$, and a five-form $\Sigma$. In particular, if $K$ is time-like we have an $S U(5)$ structure, while if $K$ is null we have a $\operatorname{Spin}(7) \ltimes \mathbb{R}^{9}$ structure. It turns out that in both cases the Killing spinor equation implies that $K$ is Killing.

The time-like case has an $S U(5)$ structure in eleven-dimensions. This structure can be demystified by noting that the time-like one-form $K$ specifies a ten-dimensional base manifold $M$ and that (essentially) the two-form $\Omega$ and $\chi$, the part of the five-form $\Sigma$ independent of $K$, satisfy the algebraic conditions of a more familiar ten-dimensional $S U(5)$ structure on $M$. The most general local form of the metric is given by

$$
d s^{2}=-\Delta^{2}(d t+\omega)^{2}+\Delta^{-1} d s^{2}\left(M_{10}\right),
$$

\footnotetext{
${ }^{2}$ See e.g. 12 for some discussion on the issue of whether or not this $G$-structure is globally defined.

${ }^{3} \mathrm{~A}$ complete classification of all supersymmetric solutions for all supergravity theories in explicit form is, of course, well beyond the scope of current techniques. For example, just consider special holonomy manifolds.
} 
where $\Delta$ is a function and $\omega$ is a one-form on the base manifold $M_{10}$. The intrinsic torsion of the $S U(5)$-structure on $M_{10}$ is only weakly constrained. Such structures have five torsion classes $W_{i}$ (see e.g. 27]). The only constraint here is that the one-form $\left.\chi\right\lrcorner d \chi$, which specifies $W_{5}$, must be equal to $12 \Delta^{-1} d \Delta$. The components of the four-form are almost determined by this data, but there is a component that isn't. To ensure that we have a solution to the equations of motion it is sufficient to impose the Bianchi identities and equations of motion for the four-form, which leads to elaborate differential conditions on $\Delta, \omega$ and the structure that still need to be solved. Note, in particular, that they constrain the part of the flux not fixed by the Killing spinor equation alone.

When $K$ is null there is a $\operatorname{Spin}(7) \ltimes \mathbb{R}^{9}$ structure. Roughly, this corresponds to being able to choose a null frame $e^{+}, e^{-}, e^{i}, e^{9}$, with $i=1, \ldots, 8$, with a $\operatorname{Spin}(7)$ structure, specified by a Cayley four-form, constructed from the $e^{i}$ only. For more details on this structure and the analysis of the Killing spinor equation leading to the most general local form of the solution, we refer to [29].

It is satisfying that such a complete description of the geometry underlying the most general supersymmetric solutions can be obtained. The final result is rather general: for example in the timelike case we noted that the geometry involves an $S U(5)$ structure in $D=10$ with only weakly constrained intrinsic torsion. But in fact this is what one might expect since the result contains all possible supersymmetric solutions preserving (at least) one time-like Killing spinor. Nevertheless, it is still useful in constructing new explicit solutions as shown in [2].

In most applications we are interested in $D=11$ supersymmetric solutions preserving more than one supersymmetry, and hence it is of interest to refine the classification and determine the conditions imposed by the presence of more supersymmetries. The classification of solutions preserving all supersymmetries in $D=10$ and $D=11$ has already been carried out some time ago [30, but the techniques used apply only to this case. However, a refinement using $G$-structures is possible. The preservation of more than one supersymmetry reduces the structure groups $S U(5)$ or $\operatorname{Spin}(7) \ltimes \mathbb{R}^{9}$ further and this is a useful tool in refining the classification. Indeed following the suggestion in [2] and the work [31] this approach is being pursued in [32, 33, 34]. It is clear that much progress can be made in this direction. It should be noted, however, that there will be many cases which will have an identity structure and the technology of $G$-structures will then not be particularly helpful in organising the calculation.

It would be very interesting if a detailed understanding of supersymmetric solutions preserving, for example, more than $1 / 2$ supersymmetry can be obtained. New solutions, supplementing the known examples of Gödel spacetimes and pp-waves, are likely to have interesting applications in M-theory. An interesting result is that backgrounds with more than 24 supersymmetries are locally homogeneous 35 .

A similar analysis for type IIB supergravity is desirable and some nice results using $G$-structure techniques have just appeared in [36].

\section{Lower-Dimensional Supergravities}

Similar techniques have also been used to study supergravity theories in lower dimensions. The major motivation for studying these theories is that they provide powerful ways of obtaining solutions relevant to string/M-theory after uplifting them to $D=10 / D=11$ supergravity. The simplicity of these supergravity theories, compared to those in $\mathrm{D}=10$ and 11, allows one to be much more explicit about the geometry. The first analysis of this 
kind was first carried out by Tod in the context of four-dimensional supergravity [37, 38, Here we will illustrate the analysis with a discussion of minimal $D=5$ supergravity [3]. The results have led to interesting new classes of explicit solutions including the surprising discovery of the maximally supersymmetric Gödel spacetime and the supersymmetric black rings, the latter to be reviewed in section 7 .

\section{1 $\mathrm{D}=5$ Minimal Supergravity}

The bosonic fields of $D=5$ minimal supergravity consist of a metric and a vector potential with field strength $F$. A bosonic solution to the equations of motion is supersymmetric if it admits a super-covariantly constant spinor obeying

$$
\left[D_{\alpha}+\frac{1}{4 \sqrt{3}}\left(\gamma_{\alpha}^{\beta \gamma}-4 \delta_{\alpha}^{\beta} \gamma^{\gamma}\right) F_{\beta \gamma}\right] \epsilon^{a}=0
$$

where $\epsilon^{a}$ is a commuting symplectic Majorana spinor.

From a single commuting spinor $\epsilon^{a}$ we can construct a scalar $f$, a 1-form $V$ and three 2 -forms $X^{(a)}$. The Clifford algebra implies that these tensors satisfy various algebraic conditions corresponding to a $G$-structure. As in $D=11$ supergravity there are two types of supersymmetric solutions to consider. When $V$ is time-like, $f, V, X$ specify an $S U(2)$ structure, while when $V$ is null they specify an $\mathbb{R}^{3}$ structure.

The next step in the classification programme is to analyse the differential conditions imposed on these structures arising from (6). After a detailed analysis one derives a local form of the most general supersymmetric solutions. Let us summarise the conditions for the time-like case. We find that the metric can be written as

$$
d s^{2}=-f^{2}(d t+\omega)^{2}+f^{-1} d s^{2}\left(M_{4}\right),
$$

where $M_{4}$ is an arbitrary hyper-Kähler space (this is the analogue of $M_{10}$ having a certain $S U(5)$ structure in the $D=11$ case), and $f$ and $\omega$ are a scalar and a one-form on $M_{4}$, respectively. The two-form field strength is given by

$$
F=\frac{\sqrt{3}}{2} d[f(d t+\omega)]-\frac{1}{\sqrt{3}} G^{-},
$$

where $G^{ \pm} \equiv \frac{1}{2} f(d \omega \pm * d \omega)$, with $*$ the Hodge dual on $M_{4}$. In order that all of the equations of motion are satisfied $f$ and $\omega$ must satisfy

$$
d G^{+}=0, \quad \Delta f^{-1}=\frac{4}{9}\left(G^{+}\right)^{2},
$$

where $\Delta$ is the Laplacian on $M_{4}$. We refer to [3] for the analogous result for the null case.

This formalism has been effectively used to construct many new solutions. One important point to emphasise is that we can obtain sensible solutions from pathological hyper-Kähler base spaces. For example, we can obtain $A d S_{2} \times S^{3}$ using a singular EguchiHanson type base, while the Gödel solution can be constructed from a singular negative mass Taub-NUT base space.

The black ring solutions, to be reviewed later, have base space $\mathbb{R}^{4}$. For such a base the above equations are actually linear if they are solved in the correct order and this can be used to construct the black ring solutions 9. We adopt a different and very effective approach [3] by first writing the metric for $\mathbb{R}^{4}$ in Gibbons-Hawking form:

$$
d s^{2}=H\left[d r^{2}+r^{2}\left(d \theta^{2}+\sin ^{2}(\theta) d \phi^{2}\right)\right]+H^{-1}(d \psi+\cos \theta d \phi)^{2}
$$


with $H=1 / r$. We will further demand that the tri-holomorphic vector field $\partial_{\psi}$ is a Killing vector of the five-dimensional metric. The significance of this is that the most general solution is then specified by three further harmonic functions, $K, L$ and $M$ on $\mathbb{R}^{3}$ (with coordinates $(r, \theta, \phi))$. In particular the general solution has

$$
\begin{aligned}
f^{-1} & =H^{-1} K^{2}+L \\
\omega & =\left(H^{-2} K^{3}+\frac{3}{2} H^{-1} K L+M\right)(d \psi+\cos \theta d \phi)+\hat{\omega}_{i} d x^{i}
\end{aligned}
$$

with $\boldsymbol{\omega}$ obtained by solving $\nabla \times \hat{\boldsymbol{\omega}}=H \nabla M-M \nabla H+\frac{3}{2}(K \nabla L-L \nabla K)$.

\subsection{Generalisations}

Similar classifications have now been carried out for a number of different supergravity theories in four [37, 38, 39, 40], five [3, 41, 49], six [42, 43] and seven [4] dimensions. Both gauged and ungauged theories have been considered as well as the possibility of including various matter fields. Although these investigations are extensive, they are not exhaustive and further theories could be considered. Furthermore, while we understand the cases of preservation of minimal and maximal supersymmetry (see also [45, 46, 47]), we would like to refine the classification to consider the cases in between, just as in the $D=11$ case. An investigation for the case of $D=7$ has been initiated in 31.

It should also be profitable to continue to seek new explicit solutions using the formalism. For example, an interesting class of asymptotically $A d S$ black holes were found in gauged supergravity in [48, 49]. Following the discovery of black rings, it seems plausible that asymptotically $A d S$ black rings also exist. It would also be very interesting to construct supersymmetric black holes with other non-spherical topologies, or alternatively prove that they do not exist by generalising the results in [50, 51].

\section{Compactifications for Phenomenology and AdS/CFT}

$G$-structures can also be used to classify particular classes of geometries of physical interest in string theory. For example, one can study warped compactifications from $\mathrm{D}=10,11$ to $d=4$ Minkowski spacetime $\left(\mathbb{M}_{4}\right)$ for model building, or to $A d S$ spaces for AdS/CFT applications. This area was recently reviewed in [12] which contains further discussion.

A warped compactification has a metric of the form

$$
d s^{2}=e^{2 \Delta} d s^{2}\left(M_{d}\right)+d s^{2}(X)
$$

where $d s^{2}\left(M_{d}\right)$ is a metric on $\mathbb{M}_{d}$ or $A d S_{d}, \Delta$ is function on the internal space $X$ with metric $d s^{2}(X)$. The significance of this ansatz is that it preserves all of the isometries of $M_{d}$. An interesting observation is that if we write the $A d S_{d}$ metric in Poincaré coordinates:

$$
d s^{2}=e^{-2 m r} d s^{2}\left(\mathbb{M}_{d-1}\right)+d r^{2}
$$

then, locally, we can view the $A d S_{d}$ cases as special examples of the warped compactifications to $\mathbb{M}_{d-1}$ by thinking of the radial coordinate as a coordinate on the internal space.

Studies of compactifications to $\mathbb{M}_{4}$ with fluxes were initiated long ago in [52, 53]. More recently these results were rederived using $G$-structures in [54, 27. General compactifications to $\mathbb{M}_{4}$ with fluxes have been studied using $G$-structures for $\mathrm{D}=11$ in [55, 56, 
57, 58, 59, for type IIB in [60, 61] and for massive IIA in 62]. Investigations into mirror symmetry were carried out in 63. In this area, it is too much to hope for explicit compact solutions ${ }^{4}$. After all recall that no explicit compact Calabi-Yau three-folds are known. Progress in the study of Calabi-Yau manifolds stem from the powerful results on the existence of Calabi-Yau metrics arising from Calabi's theorem. It would be most desirable to have analogous existence theorems for the geometries arising when the fluxes are non-vanishing building on the $G$-structure results. It seems likely that this will involve hard analysis.

On the other hand the classification of $A d S$ compactifications has been very fruitful for constructing new explicit solutions. The classification of the most general $A d S_{5}$ geometries in $\mathrm{D}=11$ supergravity produced an infinite number of new solutions [5]. Furthermore, after dimensional reduction and T-duality, a sub-class led to the discovery of an infinite new set of Sasaki-Einstein manifolds $Y^{p, q}$ which give rise to IIB $A d S_{5}$ solutions [6]. Moreover these were generalised to arbitrary odd dimensions in [65], and generalised further in [12, 66], the $D=7$ examples giving new $A d S_{4}$ solutions in $D=11$.

It seems likely that similar general classifications of $A d S_{5}$ geometries in type IIB or $A d S_{4}$ in $\mathrm{D}=11$ or type IIB will lead to the discovery of new classes of explicit solutions. A study for $A d S_{4}$ in type II appears in [67] and $A d S_{3}$ in $D=11$ appears in [68. Refining such classifications should also be a profitable undertaking. The refinement of the classification of the $A d S_{5}$ geometries arising in $D=11$ supergravity to have $N=2$ supersymmetry was presented in 44. Interestingly, the result in 4 was obtained by analytically continuing an ansatz for a class of solutions describing 1/2 BPS excitations in $A d S_{7} \times S^{4}$ and $A d S_{4} \times S^{7}$. A similar analysis of $1 / 2$ BPS excitations of $A d S_{5} \times S^{5}$ was also undertaken and a very rich set of explicit solutions were obtained. Generalising these results to situations with less supersymmetry may also be rewarding.

\section{$7 \quad \mathrm{D}=5$ Supergravity and Black Rings}

We now review the construction of the new supersymmetric black ring solutions.

\subsection{Minimal $\mathrm{D}=5$ supergravity}

The single supersymmetric black ring solution of minimal $D=5$ supergravity was found [7] by directly solving the equations (9) for a flat base space $\mathbb{R}^{4}$. The solution was rederived in [8] by writing $\mathbb{R}^{4}$ in Gibbons-Hawking form, (10), and then using equations (11). This immediately leads to a straightforward construction of multi-concentric black rings as we now discuss.

The three harmonic functions $K, L$ and $M$ in (11) for a single back ring can all be expressed in terms of a single harmonic function $h_{1}$ on $\mathbb{R}^{3}$ given by

$$
h_{1}=\frac{1}{\left|\mathbf{x}-\mathbf{x}_{1}\right|}
$$

with a single centre on the negative $z$-axis: $\mathbf{x}_{1} \equiv\left(0,0,-R^{2} / 4\right)$. Specifically:

$$
K=-\frac{q}{2} h_{1}, \quad L=1+\frac{Q-q^{2}}{4} h_{1}, \quad M=\frac{3 q}{4}-\frac{3 q R^{2}}{16} h_{1}
$$

\footnotetext{
${ }^{4}$ Non-compact solutions have important applications in gauge/gravity dualities as in e.g. 64]. See [27].
} 
and it is simple to obtain an expression for $\omega$ in (11). The solution is asymptotically flat ${ }^{5}$. It appears singular at $\mathbf{x}=\mathbf{x}_{1}$ and $\mathbf{x}=\mathbf{0}$ (recall $H=1 /|\mathbf{x}|$ in (10)), but both of these are coordinate singularities. The key to the geometry lies at $\mathbf{x}=\mathbf{x}_{1}$ which turns out to correspond to the event horizon of the black ring with topology $S^{1} \times S^{2}$. The radius of the $S^{2}$ is $q / 2$ and that of the $S^{1}$ is $l$ defined by

$$
l \equiv \sqrt{\frac{3\left(Q-q^{2}\right)^{2}}{4 q^{2}}-3 R^{2}}
$$

Reality of $l$ ensures that the geometry is free from closed-time-like curves [7].

The solution depends on three parameters $q, Q$ and $R$ which are uniquely specified by the three independent conserved charges: the mass and two angular momentum $J_{1}$ and $J_{2}$ (in five dimensions black holes can rotate in two two-planes) with $J_{1}-J_{2} \propto q R^{2}$. Supersymmetry implies that the total electric charge is not an independent charge but is specified by the mass. Note that this uniqueness property is not shared by the black ring solutions of the more general supergravity theories discussed in the next sub-section.

It is interesting to observe [7] that upon setting $R=0$, so that all four harmonic functions $H, K, L$ and $M$ have the same single centre at $\mathbf{x}=\mathbf{0}$, we recover the black hole solution of [70, 71] which has a horizon with $S^{3}$ topology and $J_{1}=J_{2}$.

Having found the single black ring solution in this language, the construction of multi concentric black rings is very simple [8]. The three harmonic functions $K, L$ and $M$ are now multi-centred:

$$
K=-\frac{1}{2} \sum_{i=1}^{N} q_{i} h_{i}, \quad L=1+\frac{1}{4} \sum_{i=1}^{N}\left(Q_{i}-q_{i}{ }^{2}\right) h_{i}, \quad M=\frac{3}{4} \sum_{i=1}^{N} q_{i}-\frac{3}{4} \sum_{i=1}^{N} q_{i}\left|\mathbf{x}_{i}\right| h_{i}
$$

with $h_{i}=1 /\left|\mathbf{x}-\mathbf{x}_{i}\right|$. The solution is parametrised by $\mathbf{x}_{i}, Q_{i}$ and $q_{i}$. The solution is fully specified after solving for $\omega$ in (11), which is not straightforward in general. However, it is straightforward when all of the poles are located on the $z$-axis, when the solution inherits another Killing symmetry. In general, the apparent singularities at the centres $\mathbf{x}_{i}$ each correspond to Killing horizons with topology $S^{1} \times S^{2}$. Thus the solutions correspond to multi black rings. For the ith ring, the radius of the $S^{2}$ is $q_{i} / 2$ and that of the $S^{1}$ is $l_{i}\left(Q_{i}, q_{i}, \mathbf{x}_{i}\right)$, the obvious generalisation of (16) $)$.

The solutions are invariant under the action of $\partial_{\psi}$ and the $S^{1}$ direction of each horizon lies on an orbit of this vector field. To see that the solutions describe concentric black rings, it is helpful to consider the orbits of $\partial_{\psi}$ in $\mathbb{R}^{4}$, which, roughly, can be thought of as the $\mathbb{R}^{4}$ at asymptotic infinity in the solution. In particular, the location of the pole in $\mathbb{R}^{3}$ for a given ring corresponds to a two-plane in $\mathbb{R}^{4}$ in which the $S^{1}$ of the ring lies. Moreover, all of these $S^{1} \mathrm{~s}$ are concentric. In the simple case that all of the poles lie in a given direction, for example the negative $z$-axis, all of the $S^{1} \mathrm{~s}$ lie in the same two-plane. While if the poles lie on a single line in $\mathbb{R}^{3}$, for example the $z$-axis, then the black rings lie in one of two orthogonal two-planes. Finally, our solutions also allow for the possibility of a single black hole being located at the common centre of all of the rings, by simply choosing one of the centres $\mathbf{x}_{i}$ to be $\mathbf{0}$. It is an interesting open question as to whether or not there are more general multi-black ring solutions that are not concentric.

By restricting to solutions with all of the poles located along the $z$-axis, we can analyse the geometry in more detail. Consider, therefore, the general solution (17) with $\mathbf{x}_{\mathbf{i}}=$ $\left(0,0,-k_{i} R_{i}^{2} / 4\right)$ and $k_{i}= \pm 1$. One finds that in order to eliminate Dirac-Misner strings,

\footnotetext{
${ }^{5}$ The generalisation that is asymptotically Gödel appears in 69 .
} 
which would lead to closed time-like curves, it is necessary to impose $\Lambda \equiv \Lambda_{1}=\Lambda_{2}=\ldots$, where $\Lambda_{i} \equiv\left(Q_{i}-q_{i}^{2}\right) / 2 q_{i}$. In this case, the radii of the rings are given by

$$
l_{i} \equiv \sqrt{3\left[\Lambda^{2}-R_{i}^{2}\right]} .
$$

This expression implies that we can place the poles anywhere on the $z$-axis provided that

$R_{i}^{2}<\Lambda^{2}$. It is interesting to observe that as the location of the pole goes to larger values of $|z|$, i.e. as $R_{i}$ increases, the circumference of the rings get uniformly smaller, perhaps contrary to one's intuition.

Another interesting observation is that there are configurations of two (say) black rings with the same asymptotic charges as the single black hole solution of [70, 71]. For example, by taking one pole to lie on the positive $z$-axis and another to lie on the negative $z$-axis one can obtain $J_{1}=J_{2}$, just as for the black hole. Moreover, and surprisingly, it is possible to choose the parameters such that the sum of the areas of the horizons of the two black rings is greater than, less than, or equal to the area of the black hole. In particular, it is possible for the black rings to be entropically preferred.

This should be contrasted with supersymmetric black holes with spherical topology. For example, in $D=4$, extreme Reissner-Nordstrom black holes with $M=|Q|$ have areas which scale as $M^{2}$. Since $M_{1}^{2}+M_{2}^{2}<\left(M_{1}+M_{2}\right)^{2}$ the entropy of the multi-black holes is less than that of a single black hole with the same total charge and mass. It will be interesting to see how the contrasting property of the rings is accounted for from a microscopic state counting point of view.

\section{$7.2 \quad \mathrm{D}=5$ supergravity coupled to vector multiplets}

It is interesting to generalise the black ring solutions just described to solutions of minimal $D=5$ supergravity coupled to an arbitrary number of vector multiplets as this gives important new solutions in string/M-theory. Recall, for example, that these supergravity theories arise as part of the low-energy effective action of M-theory reduced on a CalabiYau three fold. An interesting special case is the so-called $S T U$ model which has two vector multiplets and hence three vector fields in total. This model can arise from Mtheory reduced on a six-torus and also from type IIB supergravity reduced on a fivetorus. In the latter context, the three-charge black hole of this model (with spherical topology) then uplifts to a $D 1, D 5$, momentum system which is the setting for a precise understanding of the black hole entropy [72, 70].

The construction of the single black ring solution of these more general supergravity theories was carried out in 9, 10, 11. The construction pursued in [11 exactly mimics the construction described above for the minimal theory. One first utilises the classification of the most general supergravity solutions carried out in 49. Actually, gauged supergravity was considered in [49] but the results can easily be adapted to the ungauged case of interest here. As for minimal supergravity, there are timelike solutions and null solutions, and the black rings lie in the time-like case. The time-like solutions again have a hyper-Kähler base space and in the special case that it is of Gibbons-Hawking form, it was shown in [1] that, for the case of minimal supergravity coupled to $n-1$ vector multiplets, the solution is determined by $2 n+2$ harmonic functions in $\mathbb{R}^{3}$ (one of which specifies the hyper-Kähler manifold).

Using these results one constructs the black ring solution exactly as before using a flat hyper-Kähler base space. Furthermore, it is simple to generalise this to multi-concentric black rings with the optional possibility of a black hole (of the type [70, 73]) at the centre. 
Let us close with a brief comment about the single black ring solution of the STU model. It is specified by seven parameters $Q_{i}, q_{i}$ and $R$, where $i=1,2,3$ is an index for the three vector fields present in the model. On the other hand the solution only carries five independent conserved charges: three electric charges (which determine the mass) and two angular momentum. Thus, in contrast to the single ring of the minimal theory, the solution is not uniquely determined by the conserved charges. It will be interesting to see how this property is accounted for from a microscopic point of view. An important observation is that the extra parameters correspond to dipole charges carried by the system and this leads to an identification of the system in terms of branes and dipole branes [9, 10] and to a microscopic identification of the entropy [21] (see also [20]).

\section{Conclusions}

I hope to have given the impression that while much has been achieved in classifying supersymmetric solutions of supergravity theories, I think that much remains to be discovered.

Acknowledgement I would like to thank Jan Gutowski, Chris Hull, Dario Martelli, Stathis Pakis, Harvey Reall, James Sparks and Dan Waldram for very enjoyable collaborations upon which this review is based.

\section{References}

[1] J. P. Gauntlett, D. Martelli, S. Pakis and D. Waldram, "G-structures and wrapped NS5-branes," Commun. Math. Phys. 247, 421 (2004) arXiv:hep-th/0205050.

[2] J. P. Gauntlett and S. Pakis, "The geometry of $D=11$ Killing spinors," JHEP 0304, 039 (2003) arXiv:hep-th/0212008;

[3] J. P. Gauntlett, J. B. Gutowski, C. M. Hull, S. Pakis and H. S. Reall, "All supersymmetric solutions of minimal supergravity in five dimensions," Class. Quant. Grav. 20, 4587 (2003) arXiv:hep-th/0209114;

[4] H. Lin, O. Lunin and J. Maldacena, "Bubbling AdS space and 1/2 BPS geometries," arXiv:hep-th/0409174.

[5] J. P. Gauntlett, D. Martelli, J. Sparks and D. Waldram, "Supersymmetric $A d S_{5}$ solutions of M-theory," Class. Quant. Grav. 21, 4335 (2004) arXiv:hep-th/0402153.

[6] J. P. Gauntlett, D. Martelli, J. Sparks and D. Waldram, "Sasaki-Einstein metrics on $S^{2} \times S^{3}, "$ arXiv:hep-th/0403002.

[7] H. Elvang, R. Emparan, D. Mateos and H. S. Reall, "A supersymmetric black ring," Phys. Rev. Lett. 93 (2004) 211302 arXiv:hep-th/0407065.

[8] J. P. Gauntlett and J. B. Gutowski, "Concentric black rings," Phys. Rev. D71 (2005) 025013 arXiv:hep-th/0408010.

[9] I. Bena and N. P. Warner, "One ring to rule them all ... and in the darkness bind them?," arXiv:hep-th/0408106.

[10] H. Elvang, R. Emparan, D. Mateos and H. S. Reall, "Supersymmetric black rings and three-charge supertubes," arXiv:hep-th/0408120. 
[11] J. P. Gauntlett and J. B. Gutowski, "General concentric black rings," arXiv:hep-th/0408122,

[12] J. P. Gauntlett, D. Martelli, J. Sparks and D. Waldram, "Supersymmetric AdS backgrounds in string and M-theory," arXiv:hep-th/0411194.

[13] D. Martelli and J. Sparks, "Toric geometry, Sasaki-Einstein manifolds and a new infinite class of AdS/CFT duals," arXiv:hep-th/0411238.

[14] S. Benvenuti, S. Franco, A. Hanany, D. Martelli and J. Sparks, "An infinite family of superconformal quiver gauge theories with Sasaki-Einstein duals," arXiv:hep-th/0411264.

[15] K. Intriligator and B. Wecht, "The exact superconformal R-symmetry maximizes a," Nucl. Phys. B 667 (2003) 183 arXiv:hep-th/0304128.

[16] M. Bertolini, F. Bigazzi and A. L. Cotrone, "New checks and subtleties for AdS/CFT and a-maximization," JHEP 0412, 024 (2004) arXiv:hep-th/0411249.

[17] C. P. Herzog, Q. J. Ejaz and I. R. Klebanov, "Cascading RG flows from new SasakiEinstein manifolds," arXiv:hep-th/0412193.

[18] S. Benvenuti, A. Hanany and P. Kazakopoulos, "The toric phases of the Y(p,q) quivers," arXiv:hep-th/0412279.

[19] R. Emparan and H. S. Reall, "A rotating black ring in five dimensions," Phys. Rev. Lett. 88 (2002) 101101 arXiv:hep-th/0110260.

[20] I. Bena and P. Kraus, "Microscopic description of black rings in AdS/CFT," JHEP 0412, 070 (2004) arXiv:hep-th/0408186.

[21] M. Cyrier, M. Guica, D. Mateos and A. Strominger, "Microscopic entropy of the black ring," arXiv:hep-th/0411187.

[22] Bryant, Robert L., "Pseudo-Riemannian metrics with parallel spinor fields and vanishing Ricci tensor", Global analysis and harmonic analysis (Marseille-Luminy, 1999), 53-94, Smin. Congr., 4, Soc. Math. France, Paris, 2000.

[23] J. M. Figueroa-O'Farrill, "Breaking the M-waves," Class. Quant. Grav. 17 (2000) 2925 arXiv:hep-th/9904124.

[24] M. J. Duff and J. T. Liu, "Hidden spacetime symmetries and generalized holonomy in M-theory," Nucl. Phys. B 674, 217 (2003) arXiv:hep-th/0303140.

[25] C. N. Gowdigere, D. Nemeschansky and N. P. Warner, "Supersymmetric solutions with fluxes from algebraic Killing spinors," Adv. Theor. Math. Phys. 7, 787 (2004) arXiv:hep-th/0306097.

[26] D. D. Joyce, Compact manifolds with special holonomy, Oxford Univeristy Press, Oxford UK (2000).

[27] J. P. Gauntlett, D. Martelli and D. Waldram, "Superstrings with intrinsic torsion," Phys. Rev. D 69 (2004) 086002 arXiv:hep-th/0302158.

[28] B. de Wit, D. J. Smit and N. D. Hari Dass, "Residual Supersymmetry Of Compactified D = 10 Supergravity," Nucl. Phys. B 283, 165 (1987). 
[29] J. P. Gauntlett, J. B. Gutowski and S. Pakis, "The geometry of $D=11$ null Killing spinors," JHEP 0312, 049 (2003) arXiv:hep-th/0311112.

[30] J. Figueroa-O'Farrill and G. Papadopoulos, "Maximally supersymmetric solutions of ten- and eleven-dimensional supergravities," JHEP 0303 (2003) 048 arXiv:hep-th/0211089.

[31] O. A. P. Mac Conamhna, "Refining G-structure classifications," Phys. Rev. D 70 (2004) 105024 arXiv:hep-th/0408203.

[32] J. Gillard, U. Gran and G. Papadopoulos, "The spinorial geometry of supersymmetric backgrounds," arXiv:hep-th/0410155.

[33] M. Cariglia and O. A. P. Mac Conamhna, "Null structure groups in eleven dimensions," arXiv:hep-th/0411079.

[34] M. Cariglia and O. A. P. Mac Conamhna, "Spin(7) structures in eleven dimensions," arXiv:hep-th/0412116.

[35] J. Figueroa-O'Farrill, P. Meessen and S. Philip, "Supersymmetry and homogeneity of M-theory backgrounds," Class. Quant. Grav. 22 (2005) 207 arXiv:hep-th/0409170.

[36] U.Gran, J. Gutowski and G. Papadopoulos, "The spinorial geometry of supersymmetric type IIB backgrounds", arXiv:hep-th/0501177.

[37] K. P. Tod, "All Metrics Admitting Supercovariantly Constant Spinors," Phys. Lett. B 121 (1983) 241;

[38] K. P. Tod, "More on supercovariantly constant spinors," Class. Quant. Grav. 12 (1995) 1801.

[39] M. M. Caldarelli and D. Klemm, "All supersymmetric solutions of $\mathcal{N}=2, D=4$ gauged supergravity," JHEP 0309, 019 (2003) arXiv:hep-th/0307022;

[40] S. L. Cacciatori, M. M. Caldarelli, D. Klemm and D. S. Mansi, "More on BPS solutions of $\mathcal{N}=2, D=4$ gauged supergravity," JHEP 0407, 061 (2004) arXiv:hep-th/0406238.

[41] J. P. Gauntlett and J. B. Gutowski, "All supersymmetric solutions of minimal gauged supergravity in five dimensions," Phys. Rev. D 68, 105009 (2003) [Erratum-ibid. D 70, 089901 (2004)] arXiv:hep-th/0304064.

[42] J. B. Gutowski, D. Martelli and H. S. Reall, "All supersymmetric solutions of minimal supergravity in six dimensions," Class. Quant. Grav. 20, 5049 (2003) arXiv:hep-th/0306235.

[43] M. Cariglia and O. A. P. Mac Conamhna, "The general form of supersymmetric solutions of $\mathcal{N}=(1,0) U(1)$ and $S U(2)$ gauged supergravities in six dimensions," Class. Quant. Grav. 21, 3171 (2004) arXiv:hep-th/0402055.

[44] M. Cariglia and O. A. P. Mac Conamhna, "Timelike Killing spinors in seven dimensions," arXiv:hep-th/0407127.

[45] A. Chamseddine, J. Figueroa-O'Farrill and W. Sabra, "Supergravity vacua and Lorentzian Lie groups," arXiv:hep-th/0306278. 
[46] B. Fiol, C. Hofman and E. Lozano-Tellechea, "Causal structure of $d=5$ vacua and axisymmetric spacetimes," JHEP 0402 (2004) 034.

[47] P. Meessen and T. Ortin, "Goedel spacetimes, Abelian instantons, the graviphoton background and other flacuum solutions," Nucl. Phys. B 684 (2004) 235 arXiv:hep-th/0401005.

[48] J. B. Gutowski and H. S. Reall, "Supersymmetric AdS(5) black holes," JHEP 0402 (2004) 006 arXiv:hep-th/0401042.

[49] J. B. Gutowski and H. S. Reall, "General supersymmetric AdS(5) black holes," JHEP 0404 (2004) 048 arXiv:hep-th/0401129.

[50] H. S. Reall, "Higher dimensional black holes and supersymmetry," Phys. Rev. D 68 (2003) 024024 [Erratum-ibid. D 70 (2004) 089902] arXiv:hep-th/0211290.

[51] J. B. Gutowski, "Uniqueness of five-dimensional supersymmetric black holes," JHEP 0408 (2004) 049 arXiv:hep-th/0404079.

[52] A. Strominger, "Superstrings With Torsion," Nucl. Phys. B 274, 253 (1986).

[53] C. M. Hull, "Superstring Compactifications With Torsion And Space-Time Supersymmetry," in Turin 1985, Proceedings, Superunification and Extra Dimensions, 347375; "Compactifications Of The Heterotic Superstring," Phys. Lett. B 178 (1986) 357.

[54] G. L. Cardoso, G. Curio, G. Dall'Agata, D. Lust, P. Manousselis and G. Zoupanos, "Non-Kähler string backgrounds and their five torsion classes," Nucl. Phys. B 652 (2003) 5 arXiv:hep-th/0211118.

[55] K. Behrndt and C. Jeschek, "Fluxes in M-theory on 7-manifolds and G-structures," JHEP 0304, 002 (2003) arXiv:hep-th/0302047.

[56] P. Kaste, R. Minasian and A. Tomasiello, "Supersymmetric M-theory compactifications with fluxes on seven-manifolds and G-structures," JHEP 0307 (2003) 004.

[57] K. Behrndt and C. Jeschek, "Fluxes in M-theory on 7-manifolds: G-structures and superpotential," Nucl. Phys. B 694, 99 (2004) arXiv:hep-th/0311119.

[58] A. Lukas and P. M. Saffin, "M-theory compactification, fluxes and $A d S_{4}$," arXiv:hep-th/0403235.

[59] K. Behrndt and C. Jeschek, "Fluxes in M-theory on 7-manifolds: $G_{2}, S U(3)$ and $S U(2)$ structures," arXiv:hep-th/0406138.

[60] G. Dall'Agata, "On supersymmetric solutions of type IIB supergravity with general fluxes," Nucl. Phys. B 695, 243 (2004) arXiv:hep-th/0403220.

[61] A. R. Frey, "Notes on SU(3) structures in type IIB supergravity," JHEP 0406 (2004) 027 arXiv:hep-th/0404107.

[62] K. Behrndt and M. Cvetic, "General $\mathcal{N}=1$ supersymmetric flux vacua of (massive) type IIA string theory," arXiv:hep-th/0403049.

[63] S. Gurrieri, J. Louis, A. Micu and D. Waldram, "Mirror symmetry in generalized Calabi-Yau compactifications," Nucl. Phys. B 654 (2003) 61 arXiv:hep-th/0211102. 
[64] J. M. Maldacena and C. Nunez, "Towards the large N limit of pure $\mathrm{N}=1$ super Yang Mills," Phys. Rev. Lett. 86 (2001) 588 arXiv:hep-th/0008001.

[65] J. P. Gauntlett, D. Martelli, J. F. Sparks and D. Waldram, "A new infinite class of Sasaki-Einstein manifolds," arXiv:hep-th/0403038.

[66] W. Chen, H. Lu, C. N. Pope and J. F. Vazquez-Poritz, "A note on Einstein-Sasaki metrics in $D \geq 7, "$ arXiv:hep-th/0411218.

[67] D. Lust and D. Tsimpis, "Supersymmetric AdS(4) compactifications of IIA supergravity," arXiv:hep-th/0412250

[68] D. Martelli and J. Sparks, "G-structures, fluxes and calibrations in M-theory," Phys. Rev. D 68, 085014 (2003) arXiv:hep-th/0306225.

[69] T. Ortin, "A note on supersymmetric Goedel black holes, strings and rings of minimal $\mathrm{d}=5$ supergravity," arXiv:hep-th/0410252.

[70] J. C. Breckenridge, R. C. Myers, A. W. Peet and C. Vafa, "D-branes and spinning black holes," Phys. Lett. B 391 (1997) 93 arXiv:hep-th/9602065.

[71] J. P. Gauntlett, R. C. Myers and P. K. Townsend, "Black holes of D = 5 supergravity," Class. Quant. Grav. 16 (1999) 1 arXiv:hep-th/9810204.

[72] A. Strominger and C. Vafa, "Microscopic Origin of the Bekenstein-Hawking Entropy," Phys. Lett. B 379 (1996) 99 arXiv:hep-th/9601029.

[73] A. H. Chamseddine and W. A. Sabra, "Metrics admitting Killing spinors in five dimensions," Phys. Lett. B 426 (1998) 36 arXiv:hep-th/9801161. 